\title{
Myeloperoxidase and High-Sensitivity C-Reactive Protein for Predicting Major Adverse Cardiovascular Events in Patients with Coronary Heart Disease
}

\author{
Chenggui Liu1* ${ }^{*}$ Linong Chen' ${ }^{1}$ Yinzhong Yang², Cheng Huang1, Jun Luo ${ }^{2}$, Duanliang Peng² \\ ${ }^{1}$ Department of Clinical Laboratory, Chengdu Women's and Children's Central Hospital, Chongqing Medical \\ University, Chengdu, China \\ ${ }^{2}$ Department of Clinical Laboratory, Sichuan Academy of Medical Sciences \& Sichuan Provincial People's \\ Hospital, Chengdu, China \\ Email:
}

Received 13 March 2015; accepted 13 April 2015; published 17 April 2015

Copyright $@ 2015$ by authors and Scientific Research Publishing Inc.

This work is licensed under the Creative Commons Attribution International License (CC BY).

http://creativecommons.org/licenses/by/4.0/

(c) (i) Open Access

\section{Abstract}

Background: Research has shown that high-sensitivity C-reactive protein (hs-CRP) is a major inflammatory marker for prediction of acute coronary syndrome (ACS). Myeloperoxidase (MPO) also plays an important role in atherosclerosis initiation and development. In present study, the major adverse cardiovascular events (MACEs) of patients with coronary heart disease (CHD) were investigated. Methods: MPO, hs-CRP and ACS-related risk factors from 201 ACS (78 AMI and 123 UAP) and 210 non-ACS (84 SAP and 126 non-CHD) patients confirmed by coronary angiography were detected, and the data were analyzed with receiver operating characteristic (ROC) curve and Spearman's correlation coefficients. MACEs of 285 CHD patients were investigated during the 4-year period follow-up from March 2010 to May 2014. Results: The areas under ROC curve for diagnosing ACS were 0.888 (95\% CI 0.843 - 0.933) for MPO, and 0.862 (95\% CI 0.815-0.910) for hs-CRP, respectively. There were significantly correlations between MPO and hs-CRP in both ACS and non-ACS groups. Regarding to ACS patients, both MPO and hs-CRP were positively correlated with BMI, TC, TG, LDL-C and Hcy. Prospective study demonstrated that the incidences of MACEs associated significantly with elevated MPO baseline level (yes vs no, OR 7.383, 95\% CI 4.095 13.309) and high hs-CRP baseline level (yes vs no, OR 4.186, 95\% CI 2.469 - 7.097) in CHD patients. Conclusions: The present study provides the epidemiological evidence that elevated baseline MPO and hs-CRP levels are both valuable predictors of MACEs in CHD patients. MPO and hs-CRP would prompt the progression of atherosclerosis and development from SAP to ACS.

\footnotetext{
${ }^{*}$ Corresponding author.
}

How to cite this paper: Liu, C.G., Chen, L.N., Yang, Y.Z., Huang, C., Luo, J. and Peng, D.L. (2015) Myeloperoxidase and High-Sensitivity C-Reactive Protein for Predicting Major Adverse Cardiovascular Events in Patients with Coronary Heart Disease. International Journal of Clinical Medicine, 6, 262-270. http://dx.doi.org/10.4236/ijcm.2015.64033 


\section{Keywords}

\section{Myeloperoxidase, High Sensitivity C-Reactive Protein, Acute Coronary Syndrome, Coronary Heart Disease, Major Adverse Cardiovascular Events}

\section{Introduction}

Acute coronary syndrome (ACS) whose clinical spectrum consists of unstable angina pectoris (UAP) and acute myocardial infarction (AMI) carries the greatest risk of death and severe complications to patients with coronary heart disease (CHD). Early prediction of ACS is frequently a challenging task, while immediate risk stratification remains crucial for the prompt implementation of appropriate therapy in this setting. Research has confirmed that inflammation has been implicated at all stages in the development of atherosclerotic disease, from the early lipid deposition to plaque rupture. Plaque rupture represents the most important changes that underlie the transformation of stable coronary lesions into clinically unstable ones causing the ACS. Atherosclerotic plaque in the recent activation of the inflammatory response could become unstable plaque, in which a large number of inflammatory cells including activated neutrophils, macrophages, lymphocytes and other inflammatory cells focused on the fibrous cap of the vulnerable plaque shoulder [1]. The inflammatory process in the atherosclerotic artery may lead to increase the blood levels of inflammatory cytokines and other acute-phase reactants [2] [3].

Clinical and epidemiological studies have revealed that high sensitivity C-reactive protein (hs-CRP) as a marker of inflammation is a major cardiovascular risk factor as well as an independent risk factor for prediction of adverse cardiovascular events of CHD [4] [5]. Cheng et al. [6] investigated 581 patients who underwent coronary angiography for ACS or stable angina pectoris (SAP), and found that baseline CRP levels were independently associated with higher coronary plaque burden $(\mathrm{p}=0.002)$ and plaque volume $(\mathrm{p}=0.002)$ in the imaged coronary segment; CRP was also independently associated with the presence of large lesions (plaque burden $\geq$ $70 \%, \mathrm{p}=0.030$ ); cumulative incidence of 1-year major adverse cardiac events (MACEs) was 9.7\%; CRP levels > $3 \mathrm{mg} / \mathrm{L}$ were independently associated with a higher incidence of MACEs (hazard ratio 2.17, 95\% confidence interval [CI] 1.01 to 4.67, $\mathrm{p}=0.046$ ) and of all-cause mortality and ACS only (hazard ratio 3.58, 95\% CI 1.04 to 13.0, $\mathrm{p}=0.043$ ), compared with CRP levels $<1 \mathrm{mg} / \mathrm{L}$. Wang et al. [7] also reported that the serum hs-CRP concentrations of the AMI and UAP groups were significantly higher than those of the SAP and healthy people groups ( $<<0.01)$, and that of the AMI group were significantly higher than that of the UAP group $(p<0.05)$; the serum hs-CRP level could be used to determine the stability of plaque, and have some relevance in the ACS process, showing great value in judgments of ACS prognosis.

In addition, current studies have shown that myeloperoxidase (MPO) secreted directly by activated polymorphonuclear neutrophils (PMNs) and macrophages which also play the important roles in atherosclerosis initiation and development of cardiovascular disease, that is a potential marker for diagnosing and predicting ACS [8]-[10]. Yunoki et al. [11] study revealed that plasma MPO levels in UAP patients were significantly higher than those in SAP patients or in control subjects (UAP, 21.6 [16.7 - 44.6]; SAP, 19.3 [15.7 - 29.1]; control, 15.9 [14.7 - 18.7] ng/mL; $\mathrm{p}<0.0001$ ), and suggested that an imbalance between pro-oxidants and anti-oxidants may contribute to the progression of coronary plaque instability. In previous studies, we investigated the associations between MPO with each of the metabolic parameters, inflammatory markers, traditional CHD risk factors as well as life style habits and ACS from 536 CHD patients (363 ACS and 173 SAP) and 181 non-CHD patients, and provided the epidemiological evidence that elevated serum MPO activities were significantly associated with the prevalence of ACS in CHD patients, and MPO may be a potential early warning marker for ACS [12]. This prospective study was designed to investigate the major adverse cardiovascular events (MACEs) of CHD patients according to baseline MPO and hs-CRP levels.

\section{Materials and Methods}

\subsection{Study Patients}

A total number of 411 patients who were admitted to Sichuan Academy of Medical Sciences \& Sichuan Provincial People's Hospital from March 2010 to July 2010 were recruited. All enrolled patients had been checked by coronary angiography and were diagnosed to be 201 ACS (78 AMI and 123 UAP) and 210 non-ACS [84 stable 
angina pectoris (SAP) and 126 non-CHD] according to 2007 ACC/AHA guidelines, and 285 CHD patients were investigated the combined endpoints of MACEs, including recurrent angina, heart failure, AMI or re-myocardial infarction, and death, during the 4-year (48 \pm 1.58 months) period follow-up from March 2010 to May 2014. The presence of specific diseases was established by standardized diagnostic criteria combining the information obtained from self-reported history (among 86.6\% patients presenting with chest pain), medical records, and clinical examination. The following diseases were excluded in this study: old myocardial infarction, acute and chronic infections, autoimmune disease, recent surgery, cancer, liver diseases, renal insufficiency, blood diseases, malnutrition, adrenocortical and thyroid dysfunction.

This study was carried out in accordance with the Helsinki Declaration and approved by the Regional Ethics Committee. All participating subjects were explained their participation rights and written informed consent was obtained, and were asked about alcohol intake situation (yes or no, it was defined as yes at least once a week and drinking over 45 degrees of alcohol more than $200 \mathrm{~mL}$ ) and smoking habits (yes or no, non-smokers including never smoking and stop smoking more than one year). Blood pressure [systolic blood pressure (SBP) and diastolic blood pressure (DBP)], body weight and height were measured with standard techniques. Body mass index (BMI) was calculated as body weight (kg) divided by the square of height (m).

\subsection{Materials}

Instruments: Hitachi 7600 Automatic Biochemistry Analyzer (Hitachi High-Tech Instruments Co., Ltd., Japan) and Immage 800 Immunochemistry System (Beckman Coulter, Inc., USA).

Kit of MPO: reagent and calibrator (lot number: MPO 00110-2, Diazyme Laboratories, USA): the levels of calibrator were 129 and $688 \mathrm{ng} / \mathrm{mL}$, respectively. Quality control materials (lot number: MPO 100110-2, Diazyme Laboratories, USA), the levels of MPO were (117 \pm 23$)$, (265 \pm 40$)$ and (782 \pm 117$) \mathrm{ng} / \mathrm{mL}$, respectively. Reference interval of MPO was $1.9-16.0 \mathrm{U} / \mathrm{L}$ and elevated baseline MPO level was defined as MPO > 16.69 $\mathrm{U} / \mathrm{L}$ according to the optimal cut-off value of MPO receiver operating characteristic (ROC) curve.

Kit of hs-CRP: reagent and calibrator (lot number: M 001429 and M 004640, Beckman Coulter, Inc., USA), the level of calibrator was $35.5 \mathrm{mg} / \mathrm{L}$. Vigil ${ }^{\mathrm{TM}}$ Serology Control (lot number: M 911600, M 911601 and M 911602), the levels of hs-CRP were 0.89 (0.64 - 1.14) mg/L, 13.1 (8.1 - 18.1) mg/L and 46.3 (36.3 - 56.3) mg/L, respectively. Reference interval of hs-CRP was $0.2-3.0 \mathrm{mg} / \mathrm{L}$ and high baseline hs-CRP level was defined as hs-CRP > $3.16 \mathrm{mg} / \mathrm{L}$ according to the optimal cut-off value of hs-CRP ROC curve.

The reagents and matched calibrators (Beijing Strong Biotechnologies, Inc., China), and control materials of Liquid Assayed Multiqual ${ }^{\circledR}$ as well as Liquichek ${ }^{\mathrm{TM}}$ Homocysteine (Bio-Rad Laboratories, Inc., USA) were used for determining serum glucose (GLU), uric acid (UA), total cholesterol (TC), triglyceride (TG), high density lipoprotein cholesterol (HDL-C), low density lipoprotein cholesterol (LDL-C) and homocysteine (Hcy), respectively.

\subsection{Methods}

Blood was sampled in the morning after a 12 hs fasting, and after the patient had been sedentary in sitting or supine position for at least 15 mins. The blood of all subjects must be collected before the coronary angiography. Moreover, the blood of AMI patient was collected before percutaneous coronary intervention and thrombolytic treatment. Sampled blood was transferred, making it flowing down the wall of the tube, never directly squirted into the center in order to minimize the mechanical disruption or turbulence that could be result in hemolysis or activation.

The fresh serums were separated by centrifuging (3000 r/min) for 10 minutes, and a aliquot serum was used for the determination of GLU, UA, TC, TG, HDL-C, LDL-C and Hcy, respectively, and another aliquot serum was frozen and stored at $-80^{\circ} \mathrm{C}$ refrigerator till the tests of MPO and hs-CRP were performed. MPO was measured by Hitachi 7600 Automatic Biochemistry Analyzer with selective repression method. Determination of hs-CRP was based on non-competitive near-infrared particle immunoassay with Immage 800 Immunochemistry System. MPO and hs-CRP assays were done in duplicate and were repeated if difference of the second measure was $>10 \%$ or $<10 \%$ compared to the first. The average of the two measures was used in the analyses.

\subsection{Statistical Analysis}

All analyses were calculated with SPSS software version 16.0 (SPSS, Inc., Chicago, IL, USA). Skewed distribu- 
tion variables were expressed as median (first-fourth), and differences between two independent samples were compared by Mann-Whitney U rank-sum test. ROC curve analysis for diagnosing ACS was performed to obtain area under ROC curve (AUC) and optimal cut-off values of MPO and hs-CRP. The correlation coefficients between MPO and ACS-related risk factors as well as between hs-CRP and ACS-related risk factors were calculated by Spearman's analysis because MPO and hs-CRP were skewed. The ratios were compared with chisquare tests, and correlation coefficients were compared with Fisher's z-transformations. The associations [odds ratio (OR) and 95\% confidence interval (CI)] between the combined endpoints of cardiovascular events with elevated baseline levels of MPO and hs-CRP (expressed as yes-or-no) were evaluated by univariate logistic regression analysis.

\section{Results}

\subsection{Comparison of Basal Data between ACS and Non-ACS}

Basal data were shown in Table 1. Compared to patients with non-ACS, ACS patients were characterized by older ages, males, smokers, diabetes mellitus, hypertension, hypercholesterolemia, hypertriglyceridemia, overweight or obesity, elevated MPO and hs-CRP levels. There were no differences in alcohol intake between the two groups.

\subsection{AUC and Cut-Off Values of MPO and hs-CRP ROC Curves for Diagnosing ACS}

ROC curves of MPO and hs-CRP for diagnosing ACS were shown in Figure 1. The AUC of MPO and hs-CRP were 0.888 (95\% CI $0.843-0.933, \mathrm{p}<0.001)$ and 0.862 (95\% CI $0.815-0.910, \mathrm{p}<0.001)$, respectively. The optimal cut-off value (sensitivity; specificity) of MPO was $16.69 \mathrm{U} / \mathrm{L}$ (84.6\% and $83.3 \%$ ). When the optimal cut-off value was defined as $3.16 \mathrm{mg} / \mathrm{L}$ of hs-CRP, the sensitivity and specificity were $80.1 \%$ and $82.1 \%$, respectively.

Table 1. Comparison of basal data between ACS and non-ACS [median (first-fourth) or n (\%)].

\begin{tabular}{|c|c|c|c|}
\hline & $\operatorname{ACS}(n=201)$ & Non-ACS $(\mathrm{n}=210)$ & $\mathrm{p}$-value \\
\hline MPO & 189.25 (168.11 - 245.27) & $118.34(90.34$ - 145.14) & $<0.001$ \\
\hline hs-CRP & 6.17 (3.71 - 12.73) & $1.19(0.61-2.61)$ & $<0.001$ \\
\hline Age $\geq 61$ & $133(66.2)$ & $114(54.3)$ & 0.014 \\
\hline Male & $148(73.6)$ & 130 (61.9) & 0.011 \\
\hline Smokers & $76(37.8)$ & $55(26.2)$ & 0.011 \\
\hline Alcohol intake & $84(41.8)$ & $90(42.9)$ & 0.827 \\
\hline Diabetes mellitus & $69(34.3)$ & $52(24.8)$ & 0.033 \\
\hline Hypertension & $139(69.2)$ & $121(57.6)$ & 0.015 \\
\hline Hypercholesterolemia & $93(46.3)$ & $54(25.7)$ & $<0.001$ \\
\hline Hypertriglyceridemia & $62(30.8)$ & $33(15.7)$ & $<0.001$ \\
\hline Overweight or obesity & $134(66.7)$ & $114(54.3)$ & 0.010 \\
\hline \multicolumn{4}{|l|}{ Number of risk factor } \\
\hline 0 & $9(4.5)$ & $14(6.7)$ & 0.334 \\
\hline 1 & $17(8.5)$ & $68(32.4)$ & $<0.001$ \\
\hline 2 & $53(26.4)$ & 56 (26.7) & 0.945 \\
\hline$\geq 3$ & $122(60.7)$ & $72(34.3)$ & $<0.001$ \\
\hline
\end{tabular}

Hypertension: SBP $\geq 140 \mathrm{mmHg}$ or DBP $\geq 90 \mathrm{mmHg}$; hypercholesterolemia: TC $\geq 6.21 \mathrm{mmol} / \mathrm{L}$; hypertriglyceridemia: TG $\geq 2.26 \mathrm{mmol} / \mathrm{L}$; overweight or obesity: BMI $\geq 24 \mathrm{~kg} / \mathrm{m}^{2}$. Numbers of risk factor include 6 variables of smoking, diabetes mellitus, hypertension, hypercholesterolemia, hypertriglyceridemia, overweight or obesity. 


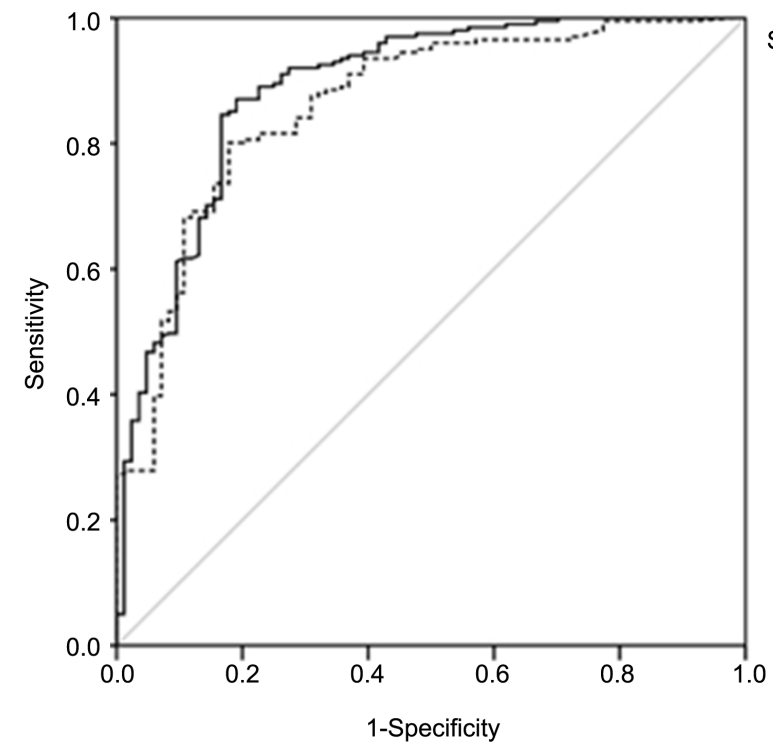

Source of the Curve

-MPO

....hSCRP

Reference Line

Figure 1. Receiver operating characteristic curves of MPO and hs-CRP for diagnosing ACS.

\subsection{The Correlations of MPO or hs-CRP with ACS-Related Risk Factors}

Table 2 showed the Spearman's correlation coefficients between MPO and hs-CRP with ACS-related risk factors. There were significantly correlations between MPO and hs-CRP ( $<0.001$ and $p=0.038$, respectively) in both ACS and non-ACS group. Regarding to ACS patients, both MPO and hs-CRP were positively correlated with BMI, TC, TG, LDL-C and Hcy ( $<<0.05$ ), and MPO were positively correlated with age, SBP and DBP (p $<0.05$ ), and negatively correlated with HDL-C levels ( $<<0.001)$, however, hs-CRP was not significantly correlated with age, SBP, DBP and HDL-C ( $>$ >0.05).

\subsection{Incidences of MACEs during the 4-Year Period Follow-Up in CHD Patients According to Baseline MPO and hs-CRP Levels}

The incidences of MACEs during the 4-year period follow-up in 285 CHD patients age 38 - 85 years according to baseline MPO and hs-CRP levels were shown in Table 3. The prospective study demonstrated that CHD patients with elevated baseline MPO levels had higher incidences of MACEs compared to CHD patients with normal-low baseline MPO levels $\left(60.87 \%\right.$ vs $\left.16.83 \%, \chi^{2}=51.041, \mathrm{p}<0.001\right)$. Similarly, elevated baseline hs-CRP levels in CHD patients had higher incidences of MACEs than normal-low baseline MPO levels (57.95\% vs $\left.24.77 \%, \chi^{2}=29.917, \mathrm{p}<0.001\right)$. There were significant associations between the combined endpoints of MACEs with elevated MPO baseline level (yes vs no, OR 7.383, 95\% CI $4.095-13.309$, p < 0.001) as well as high hs-CRP baseline level (yes vs no, OR 4.186, 95\% CI $2.469-7.097, \mathrm{p}<0.001$ ).

\section{Discussion}

ACS affects millions of individuals annually by causing considerable morbidity and mortality in both developing and developed countries. A large number of studies have demonstrated that inflammation plays a pivotal role in all phases of atherosclerosis, from the fatty streak lesion formation to the acute coronary event due to vulnerable plaque rupture [13] [14]. Inflammatory cells including PMNs and macrophages dominate early atherosclerotic lesions and vulnerable plaque, their effector molecules contribute to the pathogenesis of atherosclerosis, especially in the plaque rupture process, which are the major determinants for the ACS [15] [16].

Studies have shown that elevated baseline concentrations of hs-CRP were associated with the risk of atherosclerotic events and show a predictive value even in terms of secondary prevention, both in patients with chronic stable angina and ACS [17] [18]. Otake et al. [19] reported that levels of hs-CRP were elevated in patients with unstable angina and myocardial infarction, with high levels hs-CRP predicting worse prognosis, and there was a 
Table 2. Spearman's correlation coefficients between MPO or hs-CRP and ACS-related risk factors.

\begin{tabular}{ccccccccc}
\hline \multirow{2}{*}{ Risk factor } & \multicolumn{3}{c}{ ACS $(\mathrm{n}=201)$} & \multicolumn{3}{c}{ Non-ACS $(\mathrm{n}=210)$} \\
\cline { 2 - 9 } & MPO & p-value & hs-CRP & p-value & MPO & p-value & hs-CRP & p-value \\
\hline hs-CRP & 0.510 & $<0.001$ & & & 0.143 & 0.038 & 0.192 \\
Age & 0.150 & 0.034 & 0.101 & 0.155 & 0.087 & 0.212 & 0.090 & 0.317 \\
SBP & 0.168 & 0.017 & 0.115 & 0.103 & 0.156 & 0.023 & 0.069 & 0.571 \\
DBP & 0.195 & 0.006 & 0.080 & 0.260 & 0.164 & 0.017 & 0.039 & 0.251 \\
BMI & 0.168 & 0.017 & 0.152 & 0.031 & 0.146 & 0.035 & -0.079 & 0.251 \\
GLU & 0.114 & 0.109 & 0.101 & 0.152 & 0.034 & 0.624 & 0.080 & 0.307 \\
UA & 0.042 & 0.553 & 0.167 & 0.018 & 0.091 & 0.187 & 0.071 & 0.056 \\
TC & 0.302 & $<0.001$ & 0.292 & $<0.001$ & 0.109 & 0.115 & 0.056 & 0.421 \\
TG & 0.365 & $<0.001$ & 0.184 & 0.009 & 0.158 & 0.022 & 0.218 & 0.001 \\
HDL-C & -0.259 & $<0.001$ & -0.053 & 0.454 & -0.122 & 0.078 & -0.111 & 0.110 \\
LDL-C & 0.441 & $<0.001$ & 0.235 & 0.001 & 0.233 & 0.001 & 0.086 & 0.214 \\
Hcy & 0.168 & 0.017 & 0.175 & 0.013 & 0.046 & 0.503 & -0.004 & 0.959 \\
\hline
\end{tabular}

MPO: myeloperoxidase; hs-CRP: high-sensitivity C-reactive protein; SBP: systolic blood pressure; DBP: diastolic blood pressure; BMI: body mass index; GLU: glucose; UA: uric acid; TC: total cholesterol; TG: triglyceride; HDL-C: high density lipoprotein cholesterol; LDL-C: low density lipoprotein cholesterol; Hcy: homocysteine.

Table 3. Incidences of MACEs during the 4-year period follow-up in 285 CHD patients according to baseline MPO and hsCRP levels.

\begin{tabular}{cccccccccc}
\hline MACEs & \multicolumn{3}{c}{ Baseline MPO level } & \multicolumn{5}{c}{ Baseline hs-CRP level } \\
\hline & $\begin{array}{c}\text { Normal-low } \\
(\mathrm{n}=101)\end{array}$ & $\begin{array}{c}\text { High } \\
(\mathrm{n}=184)\end{array}$ & $\chi^{2}$ & p-value & $\begin{array}{c}\text { Normal-low } \\
(\mathrm{n}=109)\end{array}$ & $\begin{array}{c}\text { High } \\
(\mathrm{n}=176)\end{array}$ & $\chi^{2}$ & $\mathrm{p}$-value \\
\hline $\begin{array}{c}\text { Recurrent angina (\%) } \\
\text { Heart failure (\%) }\end{array}$ & 7.92 & 29.35 & 17.588 & $<0.001$ & 13.76 & 26.70 & 6.625 & 0.010 \\
$\begin{array}{c}\text { AMI or re-myocardial } \\
\text { infarction (\%) }\end{array}$ & 1.98 & 16.84 & 6.867 & 0.009 & 7.34 & 16.48 & 4.975 & 0.026 \\
Death (\%) & 0.99 & 10.32 & 6.654 & 0.010 & 2.75 & 10.23 & 5.510 & 0.019 \\
Total (\%) & 16.83 & 4.35 & 2.404 & 0.121 & 0.92 & 4.55 & 2.897 & 0.089 \\
\hline
\end{tabular}

relationship between elevated hs-CRP levels and the progression of ACS. A meta-analysis of longitudinal studies revealed that greater early blood CRP moderately increased long-term risk of recurrent cardiovascular events or death, which was a valuable prognostic predictor in patients with ACS [20].

Recent studies have also suggested that MPO is a useful risk marker and diagnostic tool in ACS and in patients admitted to emergency department for chest pain. A case-control study showed that MPO levels were significantly elevated in ACS patients compared to the patients with stable angina and the healthy control subjects [21]. Another study during a mean follow-up period of $13 \pm 4$ months in two cohorts of coronary artery disease patients (178 SAP and 130 ACS) revealed that baseline MPO level in ACS patients was an independent predictor of major adverse cardiac events during hospitalization, OR of 3.8 (95\% CI 1.2 - 12) for the combined endpoint of death, recurrent angina, heart failure, and arrhythmia [22].

The present study revealed that not only in ACS group, but also in non-ACS group, there were significantly correlations between MPO and hs-CRP. Regarding to ACS patients, both MPO and hs-CRP were positively correlated with BMI, TC, TG, LDL-C and Hcy $(\mathrm{p}<0.05)$. Patients with ACS had significantly higher levels of MPO and hs-CRP compared to patients with non-ACS ( $<<0.001)$. MPO and hs-CRP as inflammatory markers 
for diagnosing ACS, the AUC of ROC curves were 0.888 (95\% CI 0.843 - 0.933) for MPO and 0.862 (95\% CI 0.815 - 0.910) for hs-CRP, respectively. The prospective study during the 4-year period follow-up demonstrated that CHD patients with elevated baseline MPO and hs-CRP levels had higher incidences of MACEs compared to CHD patients with normal-low baseline MPO and hs-CRP levels. Elevated MPO baseline level (yes vs no, OR 7.383, 95\% CI 4.095 - 13.309) and high hs-CRP baseline level (yes vs no, OR 4.186, 95\% CI 2.469 - 7.097) were significantly associated with the combined endpoints of cardiovascular events. These findings suggest that both MPO and hs-CRP are valuable inflammatory markers for prediction of MACEs in CHD patients.

Studies have shown that CRP, an acute phase reaction protein, directly involved in atherosclerotic plaque formation and endothelial cell dysfunction [23] [24]. Firstly, CRP binding with complement C1q leads to complement pathway activation. Secondly, CRP stimulates macrophages to synthesize interleukin-6, interleukin-1 $\beta$, tumor necrosis factor- $\alpha$ and other pro-inflammatory molecules, which accelerate atherosclerotic plaque injury. Thirdly, CRP induces endothelial cells to express vascular cell adhesion molecule-1 (VCAM-1) and intercellular adhesion molecule-1 (ICAM-1), which cause monocyte migrating into arterial intima and macrophages increasing uptake of LDL.

Accumulating evidences have also implicated a role for MPO in the pathogenesis of atherosclerosis. Enriched within arterial intima and atherosclerotic plaque, MPO serves as an enzymatic source of eicosanoids and bioactive lipids and generates atherogenic forms of both LDL and HDL. For one thing, MPO has emerged as one enzymatic catalyst for LDL oxidation and carbamylation and conversion into more atherogenic high-uptake forms (oxidized-LDL and carbamylated-LDL) within the artery wall in vivo [25] [26]. For another, MPO-generated products promote the impairment of the ability of apolipoprotein A1 (apoA-1) via its site-specific modification and dysfunction of HDL, which result in the reduction of cholesterol efflux, and the increase of lipid accumulation and foam cell formation [27] [28]. Furthermore, MPO accelerates tissue damage of atherosclerotic artery and affects the transformation from stable coronary artery plaques to unstable lesions, results in ACS by oxidative stress [29].

\section{Conclusion}

The present study demonstrates that MPO levels correlate with hs-CRP levels not only in ACS group but also in non-ACS group. Regarding to ACS patients, both MPO and hs-CRP were positively correlated with BMI, TC, TG, LDL-C and Hcy. The AUCs for diagnosing ACS were 0.888 (95\% CI 0.843 - 0.933 ) for MPO, and 0.862 (95\% CI 0.815 - 0.910) for hs-CRP, respectively. Elevated baseline levels of MPO and hs-CRP are both valuable predictors of MACEs in CHD patients. MPO and hs-CRP would prompt the progression of atherosclerosis and development from SAP to ACS.

\section{Acknowledgements}

The authors would like to appreciate the staff in the Department of Clinical Laboratory and Department of Cardiovascular at the Sichuan Academy of Medical Sciences \& Sichuan Provincial People's Hospital for their support and guidance. This research was supported by the National High Technology Research and Development Program of China (863 Program) (No. 2011AA 02A 111), and Sichuan Provincial Science and Technology Department Research Foundation of China (No. 2013FZ0080), respectively. Written consent for publication was obtained from either the patients or their relatives.

\section{Study Limitations}

Since present study was just a single site study, the larger sample number of multicenter study are necessary to further confirm the value of MPO and hs-CRP for predicting major adverse cardiovascular events in CHD patients.

\section{Conflict of Interest}

The authors declare that they have no conflict of interest.

\section{References}

[1] Hansson, G.K. (2005) Inflammation, Atherosclerosis, and Coronary Artery Disease. New England Journal of Medicine, 
352, 1685-1695. http://dx.doi.org/10.1056/NEJMra043430

[2] Dominguez-Rodriguez, A., Abreu-Gonzalez, P. and Kaski, J.C. (2009) Inflammatory Systemic Biomarkers in Setting Acute Coronary Syndromes-Effects of the Diurnal Variation. Current Drug Targets, 10, 1001-1008. http://dx.doi.org/10.2174/138945009789577963

[3] Libby, P., Okamoto, Y., Rocha, V.Z. and Folco, E. (2010) Inflammation in Atherosclerosis: Transition from Theory to Practice. Circulation Journal, 74, 213-220. http://dx.doi.org/10.1253/circj.CJ-09-0706

[4] Karakas, M. and Koenig, W. (2009) CRP in Cardiovascular Disease. Herz, 34, 607-613. http://dx.doi.org/10.1007/s00059-009-3305-7

[5] Schwarzer, R., Schnell-Inderst, P., Grabein, K., Göhler, A., Stollenwerk, B., Grandi, N., et al. (2009) Prognostic Value and Clinical Effectiveness of High Sensitivity C-Reactive Protein as a Marker in Primary Prevention of Major Cardiac Events. Zeitschrift für Evidenz, Fortbildung und Qualität im Gesundheitswesen, 103, 319-329. http://dx.doi.org/10.1016/j.zefq.2009.05.020

[6] Cheng, J.M., Oemrawsingh, R.M., Garcia-Garcia, H.M., Akkerhuis, K.M., Kardys, I., de Boer, S.P., et al. (2014) Relation of C-Reactive Protein to Coronary Plaque Characteristics on Grayscale, Radiofrequency Intravascular Ultrasound, and Cardiovascular Outcome in Patients with Acute Coronary Syndrome or Stable Angina Pectoris (from the ATHEROREMO-IVUS Study). American Journal of Cardiology, 114, 1497-1503. http://dx.doi.org/10.1016/j.amjcard.2014.08.013

[7] Wang, X.H., Liu, S.Q., Wang, Y.L. and Jin, Y. (2014) Correlation of Serum High-Sensitivity C-Reactive Protein and Interleukin-6 in Patients with Acute Coronary Syndrome. Genetics and Molecular Research, 13, 4260-4266. http://dx.doi.org/10.4238/2014.June.9.11

[8] Cavusoglu, E., Ruwende, C., Eng, C., Chopra, V., Yanamadala, S., Clark, L.T., et al. (2007) Usefulness of Baseline Plasma Myeloperoxidase Levels as an Independent Predictor of Myocardial Infarction at Two Years in Patients Presenting with Acute Coronary Syndrome. American Journal of Cardiology, 99, 1364-1368. http://dx.doi.org/10.1016/j.amjcard.2006.12.060

[9] Tang, W.H., Wu, Y., Nicholls, S.J. and Hazen, S.L. (2011) Plasma Myeloperoxidase Predicts Incident Cardiovascular Risks in Stable Patients Undergoing Medical Management for Coronary Artery Disease. Clinical Chemistry, 57, 33-39. http://dx.doi.org/10.1373/clinchem.2010.152827

[10] Ferrante, G., Nakano, M., Prati, F., Niccoli, G., Mallus, M.T., Ramazzotti, V., et al. (2010) High Levels of Systemic Myeloperoxidase Are Associated with Coronary Plaque Erosion in Patients with Acute Coronary Syndromes: A Clinicopathological Study. Circulation, 122, 2505-2513. http://dx.doi.org/10.1161/CIRCULATIONAHA.110.955302

[11] Yunoki, K., Naruko, T., Inaba, M., Inoue, T., Nakagawa, M., Sugioka, K., et al. (2013) Gender-Specific Correlation between Plasma Myeloperoxidase Levels and Serum High-Density Lipoprotein-Associated Paraoxonase-1 Levels in Patients with Stable and Unstable Coronary Artery Disease. Atherosclerosis, 231, 308-314. http://dx.doi.org/10.1016/j.atherosclerosis.2013.08.037

[12] Liu, C., Xie, G., Huang, W., Yang, Y., Li, P. and Tu, Z. (2012) Elevated Serum Myeloperoxidase Activities Are Significantly Associated with the Prevalence of ACS and High LDL-C Levels in CHD Patients. Journal of Atherosclerosis and Thrombosis, 19, 435-443. http://dx.doi.org/10.5551/jat.9704

[13] Packard, R.R. and Libby, P. (2008) Inflammation in Atherosclerosis: From Vascular Biology to Biomarker Discovery and Risk Prediction. Clinical Chemistry, 54, 24-38. http://dx.doi.org/10.1373/clinchem.2007.097360

[14] Rodondi, N., Marques-Vidal, P., Butler, J., Sutton-Tyrrell, K., Cornuz, J., Satterfield, S., et al. (2010) Markers of Atherosclerosis and Inflammation for Prediction of Coronary Heart Disease in Older Adults. American Journal of Epidemiology, 171, 540-549. http://dx.doi.org/10.1093/aje/kwp428

[15] Ougrinovskaia, A., Thompson, R.S. and Myerscough, M.R. (2010) An ODE Model of Early Stages of Atherosclerosis: Mechanisms of the Inflammatory Response. Bulletin of Mathematical Biology, 72, 1534-1561. http://dx.doi.org/10.1007/s11538-010-9509-4

[16] Libby, P. (2002) Inflammation in Atherosclerosis. Nature, 420, 868-874. http://dx.doi.org/10.1038/nature01323

[17] Calabrò, P., Golia, E. and Yeh, E.T. (2009) CRP and the Risk of Atherosclerotic Events. Seminars in Immunopathology, 31, 79-94. http://dx.doi.org/10.1007/s00281-009-0149-4

[18] Arima, H., Kubo, M., Yonemoto, K., Doi, Y., Ninomiya, T., Tanizaki, Y., et al. (2008) High-Sensitivity C-Reactive Protein and Coronary Heart Disease in a General Population of Japanese: The Hisayama Study. Arteriosclerosis, Thrombosis, and Vascular Biology, 28, 1385-1391. http://dx.doi.org/10.1161/ATVBAHA.107.157164

[19] Otake, H., Shite, J., Shinke, T., Watanabe, S., Tanino, Y., Ogasawara, D., et al. (2008) Relation between Plasma Adiponectin, High-Sensitivity C-Reactive Protein, and Coronary Plaque Components in Patients with Acute Coronary Syndrome. American Journal of Cardiology, 101, 1-7. http://dx.doi.org/10.1016/j.amjcard.2007.07.041

[20] He, L.P., Tang, X.Y., Ling, W.H., Chen, W.Q. and Chen, Y.M. (2010) Early C-Reactive Protein in the Prediction of 
Long-Term Outcomes after Acute Coronary Syndromes: A Meta-Analysis of Longitudinal Studies. Heart, 96, 339346. http://dx.doi.org/10.1136/hrt.2009.174912

[21] Liang, J., Zheng, Z., Wang, M., Han, L., Zheng, Z., Peng, J., et al. (2009) Myeloperoxidase (MPO) and Interleukin-17 (IL-17) Plasma Levels Are Increased in Patients with Acute Coronary Syndromes. Journal of International Medical Research, 37, 862-866. http://dx.doi.org/10.1177/147323000903700331

[22] Roman, R.M., Camargo, P.V., Borges, F.K., Rossini, A.P. and Polanczyk, C.A. (2010) Prognostic Value of Myeloperoxidase in Coronary Artery Disease: Comparison of Unstable and Stable Angina Patients. Coronary Artery Disease, 21, 129-136. http://dx.doi.org/10.1097/MCA.0b013e328333f50d

[23] Nakou, E.S., Liberopoulos, E.N., Milionis, H.J. and Elisaf, M.S. (2008) The Role of C-Reactive Protein in Atherosclerotic Cardiovascular Disease: An Overview. Current Vascular Pharmacology, 6, 258-270. http://dx.doi.org/10.2174/157016108785909733

[24] Ferri, C., Croce, G., Cofini, V., De Berardinis, G., Grassi, D., Casale, R., et al. (2007) C-Reactive Protein: Interaction with the Vascular Endothelium and Possible Role in Human Atherosclerosis. Current Pharmaceutical Design, 13, 1631-1645. http://dx.doi.org/10.2174/138161207780831301

[25] Zhang, R., Brennan, M.L., Shen, Z., MacPherson, J.C., Schmitt, D., Molenda, C.E., et al. (2002) Myeloperoxidase Functions as a Major Enzymatic Catalyst for Initiation of Lipid Peroxidation at Sites of Inflammation. Journal of Biological Chemistry, 277, 46116-46122. http://dx.doi.org/10.1074/jbc.M209124200

[26] Wang, Z., Nicholls, S.J., Rodriguez, E.R., Kummu, O., Hörkkö, S., Barnard, J., et al. (2007) Protein Carbamylation Links Inflammation, Smoking, Uremia and Atherogenesis. Nature Medicine, 13, 1176-1184. http://dx.doi.org/10.1038/nm1637

[27] Zheng, L., Nukuna, B., Brennan, M.L., Sun, M., Goormastic, M., Settle, M., et al. (2004) Apolipoprotein A-I Is a Selective Target for Myeloperoxidase-Catalyzed Oxidation and Functional Impairment in Subjects with Cardiovascular Disease. Journal of Clinical Investigation, 114, 529-541. http://dx.doi.org/10.1172/JCI200421109

[28] Heinecke, J.W. (2007) The Role of Myeloperoxidase in HDL Oxidation and Atherogenesis. Current Atherosclerosis Reports, 9, 249-251. http://dx.doi.org/10.1007/s11883-007-0027-2

[29] Meuwese, M.C., Stroes, E.S., Hazen, S.L., van Miert, J.N., Kuivenhoven, J.A., Schaub, R.G., et al. (2007) Serum Myeloperoxidase Levels Are Associated with the Future Risk of Coronary Artery Disease in Apparently Healthy Individuals: The EPIC-Norfolk Prospective Population Study. Journal of the American College of Cardiology, 50, 159165. http://dx.doi.org/10.1016/j.jacc.2007.03.033 